\title{
Terminating the transcript: breaking up is hard to do
}

\author{
Emanuel Rosonina, ${ }^{1}$ Syuzo Kaneko, ${ }^{1}$ and James L. Manley ${ }^{2}$ \\ Department of Biological Sciences, Columbia University, New York, New York 10027, USA
}

Transcription termination occurs when a transcribing RNA polymerase releases the DNA template and the nascent RNA. Termination is required for preventing the inappropriate transcription of downstream genes, and for recycling of the polymerase. Recent studies in yeast point to the existence of gene loops, in which a transcriptionally active gene's termination region can be physically linked to its promoter site; in this situation, termination can additionally serve to facilitate transcriptional reinitiation (O'Sullivan et al. 2004; Ansari and Hampsey 2005). Termination by prokaryotic RNA polymerases, as well as eukaryotic RNA polymerase (Pol) I and Pol III, occurs at specific positions, requiring DNA or RNA cis elements that can directly or indirectly destabilize the elongating polymerase-DNA-RNA ternary complex (for reviews, see Henkin 2000; Paule and White 2000). However, for protein-encoding genes, transcribed exclusively by Pol II, transcription in most cases terminates stochastically, independently of simple cis signals, and frequently 500 or more base pairs (bp) downstream of the poly(A) signal. This unique and complex mode of termination by Pol II may have arisen to allow for transcription of very long genes without prematurely stopping at terminator-like sequences that occur by chance throughout the gene. Studies in the area of Pol II termination have sparked some debate, as two models, each supported by many studies, have emerged to explain what provokes Pol II to release the template (Fig. 1). A new study by Luo et al. (2006) presents evidence, however, that aspects of both models can be linked together and leads to new, integrated models of transcription termination.

It has been known for some time that termination is functionally connected with cleavage and polyadenylation of the nascent transcript's 3' end. In the two-step pre-mRNA 3 '-processing reaction, transcription of the poly(A) signal triggers the endonucleolytic cleavage of the nascent transcript, generating an upstream cleavage product that is immediately polyadenylated (for reviews,

\footnotetext{
${ }^{1}$ These authors contributed equally to this work.

${ }^{2}$ Corresponding author.

E-MAIL jlm2@columbia.edu; FAX (212) 865-8246.

Article and publication are at http://www.genesdev.org/cgi/doi/10.1101/ gad.1431606.
}

see Colgan and Manley 1997; Zhao et al. 1999|. The remaining downstream cleavage product, with an uncapped phosphate at its $5^{\prime}$ end, is highly unstable and is rapidly degraded (e.g., Manley et al. 1982). In mammals, the majority of protein-encoding genes contain a well conserved hexanucleotide element (AAUAAA) positioned 10-30 nucleotides (nt) upstream of the cleavage site, and a less conserved U- or GU-rich sequence $\sim 10-30$ nt downstream of the cleavage site. Multisubunit factors are responsible for recognizing these signals and catalyzing the cleavage and polyadenylation reactions. In mammals, these include the cleavage-polyadenylation specificity factor, CPSF; cleavage stimulatory factor, CstF; cleavage factors I and II, CFI and CFII; and poly(A) polymerase, PAP, which catalyzes the addition of adenosines in the poly(A) tail. Most of the components of these complexes have homologs in the yeast cleavage/ polyadenylation apparatus, which is comprised of the cleavage-polyadenylation factor, $\mathrm{CPF}$, and cleavage factors, CFIA and CFIB.

The idea of a connection between termination and cleavage/polyadenylation was first postulated in 1982 when Zaret and Sherman noticed that the aberrantly long transcripts generated from the yeast CYC1 gene lacking a 38-bp element at its $3^{\prime}$ end, reflecting defects in termination, were polyadenylated as efficiently as wildtype transcripts (Zaret and Sherman 1982). Direct support for this connection came from studies demonstrating that cleavage/polyadenylation-directing signals are also the cis elements required for normal termination in mammalian and viral systems (Whitelaw and Proudfoot 1986; Logan et al. 1987; Connelly and Manley 1988), as well as in yeast (e.g., Russo 1995). Furthermore, several yeast cleavage/polyadenylation factors are also required for normal termination, including CFIA components Rna14, Rna15, and Pcf11 (e.g., Birse et al. 1998; Sadowski et al. 2003; Kim et al. 2004a), and CPF subunits Yhh1 (Dichtl et al. 2002) and Ssu72, the latter of which is required for termination of only some genes (Ganem et al. 2003).

To address the mechanistic basis for the connection between termination and cleavage/polyadenylation, two models have emerged: the so-called "torpedo" and "allosteric" models (Fig. 1). The former model proposes that 
A
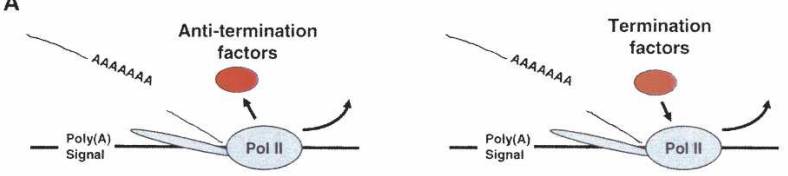

B

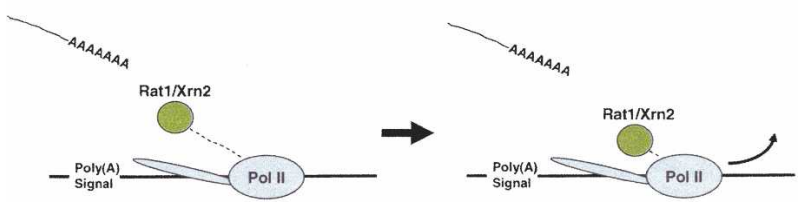

Figure 1. Models for termination by RNA Pol II. $(A)$ The allosteric model. Transcription termination is caused by the destabilization and/or a conformational change of the Pol II EC after transcribing the poly(A) site. Release of antitermination factors (left) or recruitment of termination factors (right) triggers dissociation from template DNA. $(B)$ The torpedo model. Endonucleolytic cleavage at the poly(A) site creates an entry site for the $5^{\prime} \rightarrow 3^{\prime}$ exonuclease (Rat1 in yeast and Xrn2 in humans), which degrades the RNA downstream of the cleavage site. Short nascent RNA may induce arrest of Pol II ECs and promote termination.

upon endonucleolytic cleavage of the nascent premRNA at the poly(A) site, a $5^{\prime} \rightarrow 3^{\prime}$ exonuclease degrades the downstream cleavage product while it is still tethered to Pol II. The exonuclease continues degrading the transcript until it reaches, and somehow destabilizes, the Pol II elongation complex (EC), causing termination (Connelly and Manley 1988). This model was proposed based on an analogy with Rho-dependent termination in bacteria, in which Rho factor, an RNA helicase, travels along the downstream cleavage product and destabilizes elongating RNA polymerase, resulting in termination (Nudler and Gottesman 2002). The allosteric model, also known as the antiterminator model, proposes that transcription of the poly(A) site triggers conformational changes of the EC; for example, by recruitment of a negative elongation factor or release of an antitermination factor. Such changes would destabilize the complex, resulting in termination (Logan et al. 1987). Although both models rely on recognition of the poly(A) site, a fundamental difference is that only the torpedo model depends upon the successful endonucleolytic cleavage of the nascent RNA to create the entry site for the $5^{\prime} \rightarrow 3^{\prime}$ exonuclease.

\section{The allosteric model}

A termination-inducing conformational change in the elongating Pol II complex upon transcription of the poly(A) signal might be mediated by the C-terminal domain of the largest subunit of Pol II (CTD). This repeated structure ( 25 or 26 tandem repeats in yeast, 52 in mammals) of the consensus YSPTSPS is regulated by phosphorylation throughout the transcription cycle, and is believed to coordinate events involved in the production of an mRNA, including pre-mRNA processing steps (for reviews, see Hirose and Manley 2000; Proudfoot and O'Sullivan 2002; Zorio and Bentley 2004). Expression of a truncated version of Pol II lacking the CTD demonstrated that the CTD is required for efficient cleavage and termination (McCracken et al. 1997), and considerable evidence suggests that the CTD functions in the cotranscriptional recruitment and/or assembly of protein factors involved in cleavage/polyadenylation (e.g., Hirose and Manley 1998). It is plausible that cleavage/ polyadenylation factors that interact with the CTD can mediate termination through this interaction in response to transcription of the poly(A) signal. Along these lines, yeast Pcf11, which associates directly with RNA and the CTD via its CTD-interacting domain (CID), is capable of dismantling an in vitro assembled EC (Zhang et al. 2005). Several other yeast and mammalian cleavage/polyadenylation factors can bind the CTD (McCracken et al. 1997; Barilla et al. 2001; Fong and Bentley 2001), but it is not known whether this interaction facilitates the recruitment of these factors simply for their role in cleavage and polyadenylation, or whether they are capable of transmitting signals to the EC through the CTD; for example, that the poly(A) sequence has been transcribed and recognized, or that cleavage has been successful, and termination can now take place.

Because pre-mRNA cleavage is a prerequisite for the torpedo model, support for the allosteric model of termination has come partly from experiments providing evidence that termination can apparently take place without prior cleavage. Plasmid-driven transcription in Xenopus oocyte nuclei was visualized by electron microscopy (EM), which revealed that although there is a correlation of poly(A) signal strength and termination efficiency, prior cleavage of the nascent transcript is not a prerequisite for termination (Osheim et al. 1999). In a followup study, an EM survey of $>100$ Pol II genes in Drosophila showed that whereas cotranscriptional cleavage did precede termination in some cases, in the majority the full-length transcript was released from the template prior to the cleavage event (Osheim et al. 2002). In the yeast $F B P 1$ gene, mutations in its complex poly(A) signal that strongly impair cleavage and polyadenylation have essentially no effect on termination efficiency, leading to the hypothesis that the assembly and recruitment of a partial cleavage/polyadenylation complex may suffice for termination, even though efficient cleavage and polyadenylation may not take place (Aranda et al. 1998). Supporting this notion, the cleavage/polyadenylation and termination functions of Pcf11 were shown to be separable, implying that the role of Pcfl1 in termination is not simply to promote cleavage of the transcript as an entryway for a $5^{\prime} \rightarrow 3^{\prime}$ exonuclease. The intact CID of Pcf11 is required for efficient termination in transcriptional run-on (TRO) experiments, but mutations in this domain have no effect on cleavage and polyadenylation in vitro (Sadowski et al. 2003; but see below). Conversely, whereas cells expressing the ssu72-3 temperature-sensitive (ts) mutant are defective for cleavage in vitro at the restrictive temperature, termination in these cells, as detected by TRO, is unaffected (He et al. 2003). 
Although these experiments do not necessarily exclude a role for cleavage in contributing to efficient termination, they are consistent with the notion that another signal or event, such as a conformational change of the EC, is required for termination, and in some cases might be sufficient.

Further support for the allosteric model comes from analysis of several proteins that have no direct role in cleavage/polyadenylation, yet influence elongation and/ or termination either positively or negatively. Their respective loss or recruitment might therefore trigger the speculative conformational change of the EC that is central to this model. Sub1, which is the yeast homolog of the mammalian transcriptional coactivator PC4, inhibits premature termination through its interaction with Rna15 (Calvo and Manley 2001). Cells harboring a mutation in Rna15 that increases its affinity for Sub1 show defects in termination that are suppressed by deletion of SUB1. mRNA-binding protein Npl3 also exhibits antiterminator activity, likely by competing with cleavage/polyadenylation factors for nascent RNA (Bucheli and Buratowski 2005). Release of Sub1 or Npl3 after transcription of the poly(A) signal might relieve these antiterminator activities in the EC, thus permitting termination. Factors with positive roles in termination include the G1-to-S transition-specific factor MBF component Mbp1 (Res2 in Schizosaccharomyces pombe) and Grs1, both identified through screens for 3 '-end formation defects in yeast (Magrath and Hyman 1999; Aranda and Proudfoot 2001). Despite their effects on termination, it is not known whether transcription of the poly(A) signal affects the association of these factors with the EC, or their activity, nor is it known through what mechanism they influence termination.

\section{The torpedo model}

Hallmarks of the torpedo model of termination include the requirement of prior endonucleolytic cleavage of the nascent RNA, and a $5^{\prime} \rightarrow 3^{\prime}$ exonuclease activity that can trigger template release by Pol II. A survey of the role of yeast cleavage/polyadenylation factors in termination revealed that factors involved in the cleavage reaction (Rna14, Rna15, and Pcf11), and not those involved solely in polyadenylation, are required for termination (Birse et al. 1998). A mutation in Rna15 that causes a termination defect in yeast had no effect on its RNA-binding activity, implying that it is not a defect in RNA element recognition, but an inability to catalyze cleavage, that impairs termination (Birse et al. 1998). Observations in mammalian systems of the effect of transcript length on EC processivity have lent support to the torpedo model. In vitro assembled ECs were stalled at a promoter-distal position, and the nascent transcripts were truncated to various lengths; whereas ECs associated with longer transcripts were able to resume transcription, truncation to $\sim 50 \mathrm{nt}$ or shorter resulted in the arrest of the EC (Ujvari et al. 2002). This suggests that degradation of the nascent downstream cleavage product itself might promote ar- rest of the EC, a potentially necessary step toward termination.

Strong evidence in support of the torpedo model comes from the recent identification of yeast Rat1 and human Xrn2, both $5^{\prime} \rightarrow 3^{\prime}$ RNA exonucleases, as necessary for efficient termination (Kim et al. 2004b; West et al. 2004). Rat1 is an essential protein, present at promoters and coding regions of tested genes, and most significantly at their $3^{\prime}$ ends. Despite its association with $3^{\prime}$ ends, in studies using a ts mutant allele, rat1-1, inactivation of Rat1 did not affect cleavage or polyadenylation. Similarly, RNA interference (RNAi)-mediated depletion of Xrn2 did not affect cleavage efficiency. However, chromatin immunoprecipitation (ChIP) and nuclear run-on assays revealed that both Rat1 inactivation and Xrn2 knockdown resulted in an increase in Pol II levels downstream of the poly(A) site, indicating that termination was defective. Furthermore, the downstream cleavage product was stabilized in the rat1-1 strain, as measured by RT-PCR of downstream RNA sequences, suggesting that Rat1 exonuclease activity is required for the degradation of this product (Kim et al. 2004b).

According to the torpedo model, an exonuclease activity would have to function cotranscriptionally in order to terminate transcription. Although the Rat1 exonuclease activity was not shown to be cotranscriptional, an exonuclease-inactive mutant form of Rat1 (D235A) was unable to restore termination to rat $1-1$ cells, whereas the wild-type form did rescue the defect. These results strongly suggest that the exonuclease activity of Rat 1 is required for degradation of the downstream cleavage product, and for termination of transcription by Pol II.

\section{Rat1 facilitates recruitment of $3^{\prime}$-end processing factors to the 3 ' end}

The above studies on the Rat1 and Xrn2 $5^{\prime} \rightarrow 3^{\prime}$ exonucleases showed that they are required for termination, and that their catalytic activity is critical, strongly supporting the torpedo model. But how can this be reconciled with the data suggesting that cleavage is not required for subsequent termination? Luo et al. (2006) addressed this question by examining further the role of Rat1 in termination. To do so, they introduced another $5^{\prime} \rightarrow 3^{\prime}$ exonuclease activity into the nucleus in cells where Rat 1 had been inactivated. Previously, it had been shown that fusing a nuclear localization signal (NLS) to the largely, but not exclusively, cytoplasmic yeast exonuclease Xrn1 complemented the ts phenotype of rat1-1 cells (Johnson 1997). Although the exonuclease activity provided by NLS-Xrn1 resulted in the disappearance of RNA downstream of a 3'-cleavage site, it did not rescue the termination defect in rat1-1 cells. Thus, a property of Rat1 in addition to its exonuclease activity is important for termination.

As it had been shown that a Rat1-associated protein, Rtt103, copurified with Pcf11 and other subunits of the CFIA cleavage/polyadenylation complex (Kim et al. 2004b), Luo et al. (2006) speculated that Rat1 might influence termination through interactions with the 
cleavage/polyadenylation apparatus. In support of this, ChIP analysis showed that recruitment of Rat 1 to the $3^{\prime}$ end of the ADH4 gene was reduced by Pcf11 inactivation. Reciprocally, inactivation of Ratl reduced the cross-linking of Pcf11 and Rna15 to the 3' end of ADH4. These data suggest that Rat1 is required for optimal recruitment of cleavage/polyadenylation factors that are also essential for termination. To test whether Rat1 functions in $3^{\prime}$-end processing, they tested the effect of Rat1 on the choice of alternative poly(A) sites in the ACT1 pre-mRNA. At the restrictive temperature, rat1-1 led to the preferential use of $\operatorname{distal}$ poly(A) sites versus proximal sites, a characteristic trait of mutants of $3^{\prime}$ processing factors that inhibit cleavage/polyadenylation. However, the effect was notably less severe than the shift in poly(A) site observed in strains harboring mutations in CFIA subunit Pcfl1, and whether this was a direct or indirect effect of Rat1 inactivation is not clear.

A role for Rat 1 in 3 '-end processing conflicts with evidence showing that Rat1 inactivation, or RNAi knockdown of Xrn2, has no apparent defect on poly(A) site cleavage (see above; Kim et al. 2004b; West et al. 2004). Since the poly(A) site choice assay measures the distribution of distal verses proximal cleavage/polyadenylation events, it presumably reflects the kinetics of cleavage activity, with slower kinetics favoring distal poly(A) sites. These data, therefore, suggest that Rat1 is important for optimal cleavage efficiency, perhaps by contributing to the cotranscriptional assembly of a cleavage complex. In agreement with previous results (Kim et al. 2004b), ChIP analysis showed Rat1 distributed throughout the coding region with greater accumulation at the $3^{\prime}$ end of the genes tested. These data collectively suggest a nonessential role for Rat 1 in $3^{\prime}$-end processing, and it is possible that the association of Rat 1 with 3 '-end processing factors simply facilitates its role in cotranscriptional degradation of nascent RNA by ensuring that the exonuclease is present when endonucleolytic cleavage takes place. It remains debatable, then, whether the termination defect observed upon inactivation of Ratl reflects the insufficient recruitment of cleavage/polyadenylation factors at the poly(A) site, in addition to, or instead of, loss of its exonuclease activity.

\section{Cotranscriptional degradation of nascent RNA by Rat1 and Xrn1 exonucleases}

Previous work proposed, but did not demonstrate, that Rat1 and Xrn2 degrade nascent RNA cotranscriptionally (Kim et al. 2004b; West et al. 2004). To confirm this, Luo et al. (2006) employed RNA immunoprecipitation (RIP) analysis, in which Pol II is cross-linked to the nascent RNA downstream of the poly(A) site. Detection of this RNA by RT-PCR after immunoprecipitation of Pol II indicates that it has not been degraded. Surprisingly, they found that inactivation of Rat l alone did not result in accumulation of downstream RNA. Only in rat1-1 xrn1s cells, at the restrictive temperature, was the downstream RNA detectable, indicating that both Rat1 and Xrn1 can participate in cotranscriptional degrada- tion. These data confirmed that degradation of downstream RNA is cotranscriptional, since in the presence of either Rat1 or Xrn1, the downstream RNA was not found associated with Pol II. Moreover, even though Xrn1 is able to degrade nascent downstream RNA (at least in the absence of functional Rat1), it does not function in termination (see above), indicating that degradation of the nascent RNA is not sufficient to cause termination. This is consistent with previous data showing that in some situations termination can be defective, while cleavage, and presumably exonuclease degradation of the downstream product, is functional (Magrath and Hyman 1999; Sadowski et al. 2003).

\section{Transcriptional termination by Pol II: a complex process}

Adding to the complexity of termination, sequence elements bind protein factors that are involved in Pol II termination, yet function independently of 3 '-end processing. Binding of the CCAAT box in the adenovirus major late promoter by $\mathrm{CP}-1$ is necessary for termination of transcription of the upstream gene (Connelly and Manley 1989). Similarly, a zinc finger protein, MAZ, binds a G-rich element, perhaps contributing to termination between closely spaced human complement genes C2 and factor B (Ashfield et al. 1991). Considering how DNA-binding proteins are able to interfere with Pol II transcription, pausing of the EC might play a role in facilitating termination. Indeed, pausing has been shown to be important for termination, at least for some genes (e.g., Enriquez-Harris et al. 1991). Pausing might serve to facilitate cleavage/polyadenylation (Yonaha and Proudfoot 1999), thereby indirectly promoting termination, or to slow Pol II, allowing for template release, as in the case of closely spaced genes. Pausing can depend on sequence elements (references above) or can be independent of any downstream elements in the DNA (Tran et al. 2001). Interestingly, pausing of Pol II at the MAZ4 elements was found to correlate with degradation of the nascent RNA by Xrn2, suggesting that Xrn2 can be responsible, at least in part, for pause-mediated termination (Gromak et al. 2006).

While additional elements, such as pause sites, contribute to the complexity of termination, the only sequence element generally required for termination is the poly(A) signal itself. Indeed, the strength of the poly(A) signal affects the efficiency of termination (Osheim et al. 1999; Orozco et al. 2002), suggesting that other elements play an auxiliary role. For example, they might become more important when efficient and precise control of termination is required, as in the case of the closely spaced adenovirus and complement genes mentioned above. Adding to the complexity of Pol II termination, chromatin structure across the termination region was also found to affect termination efficiency. Cells lacking the Chd1 chromatin remodeling factor showed termination defects and defects in both chromatin structure at termination regions and termination itself (Alen et al. 2002). Chromatin remodeling at sites downstream of the 
poly(A) site might be important to induce or facilitate pausing or template release by Pol II.

It is clear that the presence of $3^{\prime}$-processing factors at the poly(A) signal and consequent processes are essential for transcription termination, but what is the critical event that ensures that Pol II will release the template? The importance of the Rat1 and Xrn2 5' $\rightarrow$ 3' exonucleases indicates that a conformation change in the $\mathrm{EC}$, as proposed by the allosteric model, is insufficient to promote termination, and strongly supports the torpedo model. On the other hand, data discussed above, plus the recent evidence indicating that exonuclease digestion of the downstream RNA is not sufficient to trigger termination, argue against the torpedo model in its simplest form. To address this, Luo et al. (2006) proposed a hybrid model in which Ratl is an essential component of a Pol II complex that achieves cleavage at the poly(A) site, degradation of the nascent downstream RNA, and undergoes allosteric changes that promote its release from the template. But what these allosteric changes might be and how they would affect dissociation of the EC remain unclear.

Thus we have an apparent "termination paradox." The exonuclease activity of Rat1/Xrn2 appears to be essential for termination, yet exonuclease degradation of the nascent downstream RNA may not be. One possibility is that exonuclease activity is required in some cases but not others. For example, perhaps when a strong poly(A) signal is present, an allosteric change is sufficient to allow the EC to enter a "termination-ready" state. A strong poly(A) signal might include pause-stimulating elements and associated factors. In all other cases, achieving this termination-ready state would depend on the cotranscriptional degradation of the nascent transcript by Rat1/Xrn2 (Fig. 2). But how then do we explain the EM data that most of the observed transcripts in Drosophila nuclei appear uncleaved upon termination (Osheim et al. 2002)? One possibility is that many poly(A) sites are sufficient on their own to induce termination by allosteric modifications, thereby bypassing the requirement for exonuclease degradation of the downstream RNA. However, the EM results are not inconsistent with what we might call a "concerted torpedo" model, in which all steps-cleavage/polyadenylation, exonucleolytic degradation, and termination-occur in a coupled manner in a single complex (Fig. 2). Indeed, the finding that Rat 1 seems to function as an integral component of the cleavage complex (Luo et al. 2006) adds support for this idea.

Once the EC is in the termination-ready state, an additional signal might be required to stimulate transcript release (Fig. 2). Pcf11, or more specifically its CID, is able to dismantle paused Pol II complexes from template DNA (Zhang et al. 2005), suggesting that it might play a central role in template release. Genetic evidence first pointed to separable functions of Pcf11 in 3 '-end processing and in termination, indicating that termination defects associated with the CID were not due to defects in cleavage and polyadenylation (Sadowski et al. 2003). Evidence from Luo et al. (2006), however, shows that the

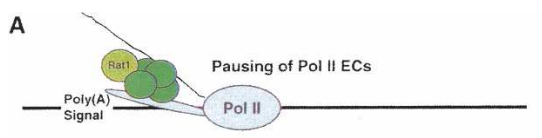

B
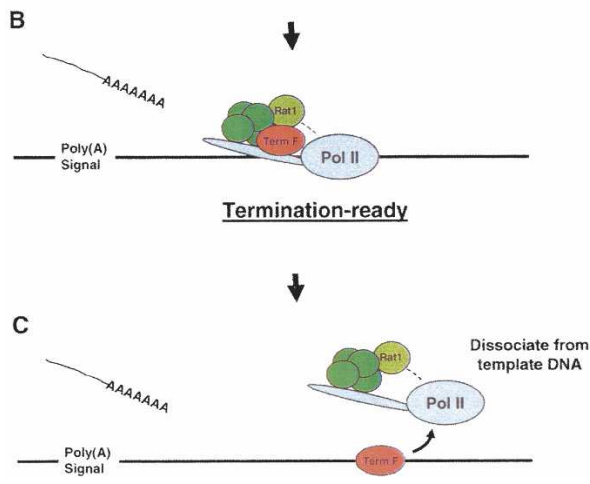

Figure 2. Concerted torpedo model for termination by RNA Pol II. (A) Immediately after passing through the poly(A) site, Pol II ECs pause transcription induced by one of several mechanisms (see text); for example, through a conformational change involving the 3 '-end processing factors (green). (B) Endonucleolytic cleavage at the polyadenylation site and subsequent degradation of 3'-cleaved RNA by Rat1/Xrn2. Simultaneously, Rat1/Xrn2 recruits termination factors (Term F) and delivers them to the Pol II active site. $(C)$ The resulting "terminationready" Pol II is targeted by Term F. RNA-binding proteins, and especially helicase-like ATPases such as TTF2 (see text), are candidates for Term F.

same Pcf11 mutation previously shown to affect only cleavage in fact causes both cleavage and termination defects. As such, it is unclear whether Pcf11 affects termination independently of its function as a 3 '-end processing factor. Significantly, if Pcf11 functions in cleavage and termination are indeed inseparable, this removes another apparent argument against the torpedo model. Thus, a parsimonious view of available data suggests that exonuclease activity is indeed necessary, but not sufficient, for termination.

What other factor might be directly responsible for dissociating Pol II ECs? Considering the evidence that termination factors, Rho in bacteria (Nudler and Gottesman 2002), NPH-I in vaccinia virus (Deng and Shuman 1998), and Sen 1 in yeast snRNA and snoRNA genes (Steinmetz et al. 2001) use ATP hydrolysis and likely act as helicases to disrupt RNA-DNA hybrids within the RNA polymerase active site, we suggest that a similar factor is required to dissociate Pol II from the template DNA of mRNA-encoding genes. Transcription termination factor 2, TTF2, is a well characterized Pol II-associated factor, with DNA-dependent ATPase activity and a helicase domain (Jiang et al. 2004), that may fit the bill. During mitosis, when Pol II transcription is transiently shut down, TTF2 translocates to the nucleus and releases nascent RNA and Pol II from the template in a reaction requiring ATP hydrolysis (Jiang et al. 2004). Although it is not known whether TTF2 functions during interphase transcription, TTF2, or a similar factor, might be recruited to stimulate template release. This could be 
by association with Pcf11 or another nascent RNA-binding factor, or, intriguingly, with the Rat1/Xrn2 complex itself. Indeed, if Rat1 or an associated protein specifically recruits a TTF2-like helicase, then this provides an explanation why Rat1 but not Xrn1 (which would be unable to recruit the helicase) is able to function in termination. Such cooperative termination activity is well documented in bacteria and vaccinia virus RNA polymerase (Deng and Shuman 1998; Nudler and Gottesman 2002). According to this scenario, the role of the exonuclease is both to degrade the RNA downstream of the cleavage site and simultaneously to deliver a helicaselike ATPase, such as TTF2, which disassembles the EC, finally releasing the nascent RNA and DNA template (Fig. 2).

The identification of the $5^{\prime} \rightarrow 3^{\prime}$ exonucleases that degrade the nascent downstream RNA cleavage product and the demonstration that they are necessary for termination has provided considerable insight into the mechanism of Pol II termination. But the finding that something in addition is required is important and opens additional possibilities. For example, neither model-the torpedo nor the allosteric-in its simplest form is sufficient to explain what signals provoke Pol II to release the template. While the torpedo model has been considerably strengthened, new integrated models have now been proposed. With these important new insights in hand, it will be interesting to see what new discoveries, such as the identification of an essential termination helicase, will be forthcoming.

\section{Acknowledgments}

We thank I. Boluk for assistance in the preparation of the manuscript. E. Rosonina is a Research Fellow of The Terry Fox Foundation through an award from the National Cancer Institute of Canada. Work in our laboratory is supported by funds from the National Institutes of Health.

\section{References}

Alen, C., Kent, N.A., Jones, H.S., O'Sullivan, J., Aranda, A., and Proudfoot, N.J. 2002. A role for chromatin remodeling in transcriptional termination by RNA polymerase II. Mol. Cell 10: $1441-1452$.

Ansari, A. and Hampsey, M. 2005. A role for the CPF 3'-end processing machinery in RNAP II-dependent gene looping. Genes \& Dev. 19: 2969-2978.

Aranda, A. and Proudfoot, N. 2001. Transcriptional termination factors for RNA polymerase II in yeast. Mol. Cell 7: 10031011.

Aranda, A., Perez-Ortin, J.E., Moore, C., and del Olmo, M.L. 1998. Transcription termination downstream of the Saccharomyces cerevisiae $\mathrm{FBP} 1$ [changed from $\mathrm{FPB} 1$ ] poly(A) site does not depend on efficient 3 ' end processing. RNA 4: 303318.

Ashfield, R., Enriquez-Harris, P., and Proudfoot, N.J. 1991. Transcriptional termination between the closely linked human complement genes $\mathrm{C} 2$ and factor B: Common termination factor for C2 and c-myc? EMBO J. 10: 4197-4207.

Barilla, D., Lee, B.A., and Proudfoot, N.J. 2001. Cleavage/poly- adenylation factor IA associates with the carboxyl-terminal domain of RNA polymerase II in Saccharomyces cerevisiae. Proc. Natl. Acad. Sci. 98: 445-450.

Birse, C.E., Minvielle-Sebastia, L., Lee, B.A., Keller, W., and Proudfoot, N.J. 1998. Coupling termination of transcription to messenger RNA maturation in yeast. Science 280: 298301.

Bucheli, M.E. and Buratowski, S. 2005. Npl3 is an antagonist of mRNA 3' end formation by RNA polymerase II. EMBO I. 24: 2150-2160.

Calvo, O. and Manley, J.L. 2001. Evolutionarily conserved interaction between CstF-64 and PC4 links transcription, polyadenylation, and termination. Mol. Cell 7: 1013-1023.

Colgan, D.F. and Manley, J.L. 1997. Mechanism and regulation of mRNA polyadenylation. Genes \& Dev. 11: 2755-2766.

Connelly, S. and Manley, J.L. 1988. A functional mRNA polyadenylation signal is required for transcription termination by RNA polymerase II. Genes \& Dev. 2: 440-452.

1989. A CCAAT box sequence in the adenovirus major late promoter functions as part of an RNA polymerase II termination signal. Cell 57: 561-571.

Deng, L. and Shuman, S. 1998. Vaccinia NPH-I, a DExH-box ATPase, is the energy coupling factor for mRNA transcription termination. Genes \& Dev. 12: 538-546.

Dichtl, B., Blank, D., Sadowski, M., Hubner, W., Weiser, S., and Keller, W. 2002. Yhh1p/Cftlp directly links poly(A) site recognition and RNA polymerase II transcription termination. EMBO J. 21: 4125-4135.

Enriquez-Harris, P., Levitt, N., Briggs, D., and Proudfoot, N.J. 1991. A pause site for RNA polymerase II is associated with termination of transcription. EMBO J. 10: 1833-1842.

Fong, N. and Bentley, D.L. 2001. Capping, splicing, and 3' processing are independently stimulated by RNA polymerase II: Different functions for different segments of the CTD. Genes \& Dev. 15: 1783-1795.

Ganem, C., Devaux, F., Torchet, C., Jacq, C., QuevillonCheruel, S., Labesse, G., Facca, C., and Faye, G. 2003. Ssu72 is a phosphatase essential for transcription termination of snoRNAs and specific mRNAs in yeast. EMBO J. 22: 15881598.

Gromak, N., West, S., and Proudfoot, N.J. 2006. Pause sites promote transcriptional termination of mammalian RNA polymerase II. Mol. Cell. Biol. (in press).

He, X., Khan, A.U., Cheng, H., Pappas Jr., D.L., Hampsey, M., and Moore, C.L. 2003. Functional interactions between the transcription and mRNA $3^{\prime}$ end processing machineries mediated by Ssu72 and Sub1. Genes \& Dev. 17: 1030-1042.

Henkin, T.M. 2000. Transcription termination control in bacteria. Curr. Opin. Microbiol. 3: 149-153.

Hirose, Y. and Manley, J.L. 1998. RNA polymerase II is an essential mRNA polyadenylation factor. Nature 395: 93-96.

- 2000. RNA polymerase II and the integration of nuclear events. Genes \& Dev. 14: 1415-1429.

Jiang, Y., Liu, M., Spencer, C.A., and Price, D.H. 2004. Involvement of transcription termination factor 2 in mitotic repression of transcription elongation. Mol. Cell 14: 375-385.

Johnson, A.W. 1997. Ratlp and Xrnlp are functionally interchangeable exoribonucleases that are restricted to and required in the nucleus and cytoplasm, respectively. Mol. Cell. Biol. 17: 6122-6130.

Kim, M., Ahn, S.H., Krogan, N.J., Greenblatt, J.F., and Buratowski, S. 2004a. Transitions in RNA polymerase II elongation complexes at the $3^{\prime}$ ends of genes. EMBO J. 23: 354-364.

Kim, M., Krogan, N.J., Vasiljeva, L., Rando, O.J., Nedea, E., Greenblatt, J.F., and Buratowski, S. 2004b. The yeast Rat1 exonuclease promotes transcription termination by RNA 
polymerase II. Nature 432: 517-522.

Logan, J., Falck-Pedersen, E., Darnell Jr., J.E., and Shenk, T. 1987. A poly(A) addition site and a downstream termination region are required for efficient cessation of transcription by RNA polymerase II in the mouse $\beta$ maj-globin gene. Proc. Nat1. Acad. Sci. 84: 8306-8310.

Luo, W., Johnson, A.W., and Bentley, D.L. 2006. The role of Rat1 in coupling mRNA $3^{\prime}$-end processing to transcription termination: Implications for a unified allosteric-torpedo model. Genes \& Dev. 20: 954-965.

Magrath, C. and Hyman, L.E. 1999. A mutation in GRS1, a glycyl-tRNA synthetase, affects 3'-end formation in Saccharomyces cerevisiae. Genetics 152: 129-141.

Manley, J.L., Sharp, P.A., and Gefter, M.L. 1982. RNA synthesis in isolated nuclei processing of adenovirus serotype 2 late messenger RNA precursors. J. Mol. Biol. 159: 581-599.

McCracken, S., Fong, N., Yankulov, K., Ballantyne, S., Pan, G., Greenblatt, J., Patterson, S.D., Wickens, M., and Bentley, D.L. 1997. The C-terminal domain of RNA polymerase II couples mRNA processing to transcription. Nature 385: 357-361.

Nudler, E. and Gottesman, M.E. 2002. Transcription termination and anti-termination in E. coli. Genes Cells 7: 755-768.

Orozco, I.J., Kim, S.J., and Martinson, H.G. 2002. The poly(A) signal, without the assistance of any downstream element, directs RNA polymerase II to pause in vivo and then to release stochastically from the template. J. Biol. Chem. 277: 42899-42911.

Osheim, Y.N., Proudfoot, N.J., and Beyer, A.L. 1999. EM visualization of transcription by RNA polymerase II: Downstream termination requires a poly(A) signal but not transcript cleavage. Mol. Cell 3: 379-387.

Osheim, Y.N., Sikes, M.L., and Beyer, A.L. 2002. EM visualization of Pol II genes in Drosophila: Most genes terminate without prior $3^{\prime}$ end cleavage of nascent transcripts. Chromosoma 111: 1-12.

O'Sullivan, J.M., Tan-Wong, S.M., Morillon, A., Lee, B., Coles, J., Mellor, J., and Proudfoot, N.J. 2004. Gene loops juxtapose promoters and terminators in yeast. Nat. Genet. 36: 1014 1018.

Paule, M.R. and White, R.J. 2000. Survey and summary: Transcription by RNA polymerases I and III. Nucleic Acids Res. 28: $1283-1298$.

Proudfoot, N. and O'Sullivan, J. 2002. Polyadenylation: A tail of two complexes. Curr. Biol. 12: R855-R857.

Russo, P. 1995. Saccharomyces cerevisiae mRNA 3' end forming signals are also involved in transcription termination. Yeast 11: 447-453.

Sadowski, M., Dichtl, B., Hubner, W., and Keller, W. 2003. Independent functions of yeast Pcfl1p in pre-mRNA 3 ' end processing and in transcription termination. EMBO J. 22: 2167-2177.

Steinmetz, E.J., Conrad, N.K., Brow, D.A., and Corden, J.L. 2001. RNA-binding protein Nrd1 directs poly(A)-independent 3 '-end formation of RNA polymerase II transcripts. $\mathrm{Na}$ ture 413: 327-331.

Tran, D.P., Kim, S.J., Park, N.J., Jew, T.M., and Martinson, H.G. 2001. Mechanism of poly(A) signal transduction to RNA polymerase II in vitro. Mol. Cell. Biol. 21: 7495-7508.

Ujvari, A., Pal, M., and Luse, D.S. 2002. RNA polymerase II transcription complexes may become arrested if the nascent RNA is shortened to less than 50 nucleotides. J. Biol. Chem. 277: 32527-32537.

West, S., Gromak, N., and Proudfoot, N.J. 2004. Human 5' $\rightarrow 3^{\prime}$ exonuclease Xrn2 promotes transcription termination at cotranscriptional cleavage sites. Nature 432: 522-525.
Whitelaw, E. and Proudfoot, N. 1986. $\alpha$-Thalassaemia caused by a poly(A) site mutation reveals that transcriptional termination is linked to $3^{\prime}$ end processing in the human $\alpha 2$ globin gene. EMBO J. 5: 2915-2922.

Yonaha, M. and Proudfoot, N.J. 1999. Specific transcriptional pausing activates polyadenylation in a coupled in vitro system. Mol. Cell 3: 593-600.

Zaret, K.S. and Sherman, F. 1982. DNA sequence required for efficient transcription termination in yeast. Cell 28: $563-$ 573.

Zhang, Z., Fu, J., and Gilmour, D.S. 2005. CTD-dependent dismantling of the RNA polymerase II elongation complex by the pre-mRNA 3 '-end processing factor, Pcf11. Genes \& Dev. 19: 1572-1580.

Zhao, J., Hyman, L., and Moore, C. 1999. Formation of mRNA $3^{\prime}$ ends in eukaryotes: Mechanism, regulation, and interrelationships with other steps in mRNA synthesis. Microbiol. Mol. Biol. Rev. 63: 405-445.

Zorio, D.A. and Bentley, D.L. 2004. The link between mRNA processing and transcription: Communication works both ways. Exp. Cell Res. 296: 91-97. 


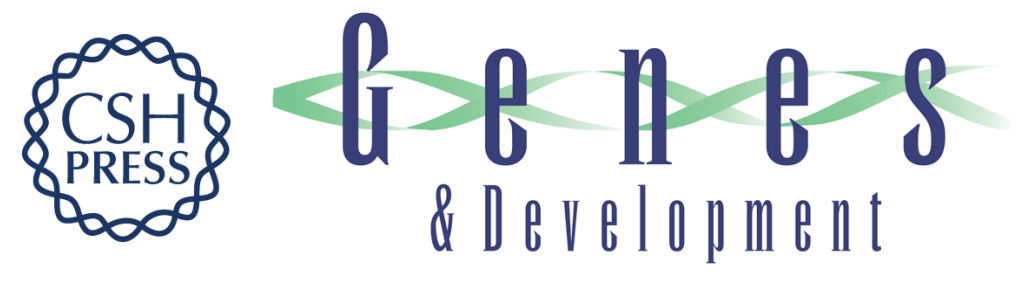

\section{Terminating the transcript: breaking up is hard to do}

Emanuel Rosonina, Syuzo Kaneko and James L. Manley

Genes Dev. 2006, 20:

Access the most recent version at doi:10.1101/gad.1431606

Related Content The role of Rat1 in coupling mRNA 32-end processing to transcription termination: implications for a unified allosterictorpedo model Weifei Luo, Arlen W. Johnson and David L. Bentley Genes Dev. UNKNOWN , 2006 20: 954-965

References This article cites 49 articles, 23 of which can be accessed free at: http://genesdev.cshlp.org/content/20/9/1050.full.html\#ref-list-1

Articles cited in:

http://genesdev.cshlp.org/content/20/9/1050.full.html\#related-urls

\section{License}

Email Alerting

Receive free email alerts when new articles cite this article - sign up in the box at the top Service right corner of the article or click here.

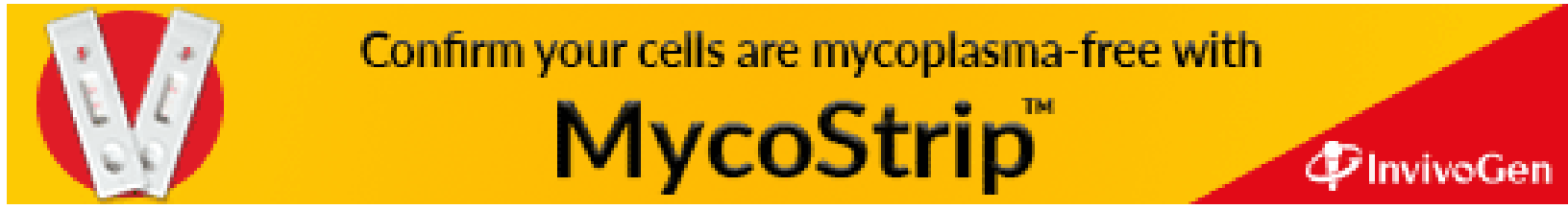

